\title{
The Adaptive Algorithm of Parallel Genetic Optimization Based on Orthogonal Wavelet of Space Diversity
}

\author{
Jiang Xiaoping, Liu Xin ${ }^{*}$, Ding Hao and Li Chenghua
}

College of Electronics and Information Engineering, Hubei Key Laboratory of Intelligent Wireless Communications, South-Central University for Nationalities, Wuhan 430074, China

\begin{abstract}
Diversity technology can effectively resist channel multipath fading, and balanced technology can effectively inhibit the intersymbol interference, so combining the diversity technology and balanced technology can effectively improve the quality of communication. This paper uses parallel adaptive genetic algorithm of orthogonal wavelet based on spatial diversity, taking the space diversity of each branch equalizer weight vector as the son species clusters of parallel genetic algorithms for selection, crossover and mutation, sending the individual of the best fitness between each son species clusters regularly, and eliminating the individual of the worst fitness, then taking diversity branch output signals equal gain combined with inputs orthogonal wavelet adaptive device. The simulation results show that the algorithm has the characters of fast convergence, small steady-state error and superior solution of global.
\end{abstract}

Keywords: Adaptive, Parallel genetic algorithm, Space diversity, The wavelet transform.

\section{INTRODUCTION}

In communication system, the channel's multipath fading and the transmission time delay phenomenon seriously affect the reliability of digital communication between transmitter and receiver. An effective method to eliminate the channel fading is adopting diversity technology, which includes frequency diversity, time diversity and space diversity. Compared with the spatial diversity, frequency diversity and time diversity will take up too much bandwidth. The adaptive technology is one of the most effective ways to overcome the inter-symbol interference, so the combination of space diversity and adaptive technique can effectively overcome channel fading and inter-symbol interference, thus improving the performance of communication system [1]. Orthogonal wavelet adaptive algorithm can reduce the correlation between signal and noise through orthogonal wavelet transform, thus accelerating the convergence speed [2]. However, initialization of an adaptive algorithm for the weight vector is sensitive; making the algorithm easily fall into local minimum value, and even divergence [3].

Genetic algorithm (GA) provides a generic framework for solving complex system optimization problems, it does not depend on the problem of specific areas, and the problem of the species has a strong robustness. However, the traditional genetic algorithm efficiency is not high and easy in premature convergence. Parallel genetic algorithm is used in traditional genetic algorithms intrinsic parallel mechanism

*Address correspondence to this author at the College of Electronics and Information Engineering, Hubei Key Laboratory of Intelligent Wireless Communications, South-Central University for Nationalities, Wuhan 430074,P.R. China; Tel: 15608656252; E-mail: 15608656252@163.com to improve the accuracy of the algorithm efficiency and the precision of the solution and to avoid premature convergence and accelerate convergence speed [4].

On the basis of above analysis, this paper will reference to the parallel genetic algorithm based on orthogonal wavelet adaptive algorithm of space diversity. By using parallel genetic algorithm for spatial diversity, equalizer weight vector of each branch is optimized, followed by the diversity branch output signal input orthogonal wavelet adaptive device after the merger. The computer simulation shows that the algorithm convergence speed is fast, with small steadystate error, and can achieve the global optimal solution.

\section{THE NORM OF THE ADAPTIVE ALGORITHM BASED ON ORTHOGONAL WAVELET TRANSFORM}

The norm of the adaptive algorithm based on orthogonal wavelet (WTCMA) principle is shown in Fig. (1).

In Fig. (1), $n$ shows the time sequence, $\boldsymbol{a}(n)$ shows the launch signal, $\boldsymbol{c}(n)$ shows the channel impulse response, $\boldsymbol{w}(n)$ shows the channel output of additive white gaussian noise, $\boldsymbol{y}(n)$ shows the equalizer input signal, $\boldsymbol{R}(n)$ shows the signal after orthogonal wavelet transform, $\boldsymbol{f}(n)$ shows the equalizer weight vector, and $z(n)$ shows the signal after equilibrium.

The norm adaptive algorithm of orthogonal wavelet transform for the cost function is given as:

$$
J(\boldsymbol{f})=E\left\{\left[R_{2}-|z(n)|^{2}\right]^{2}\right\}
$$




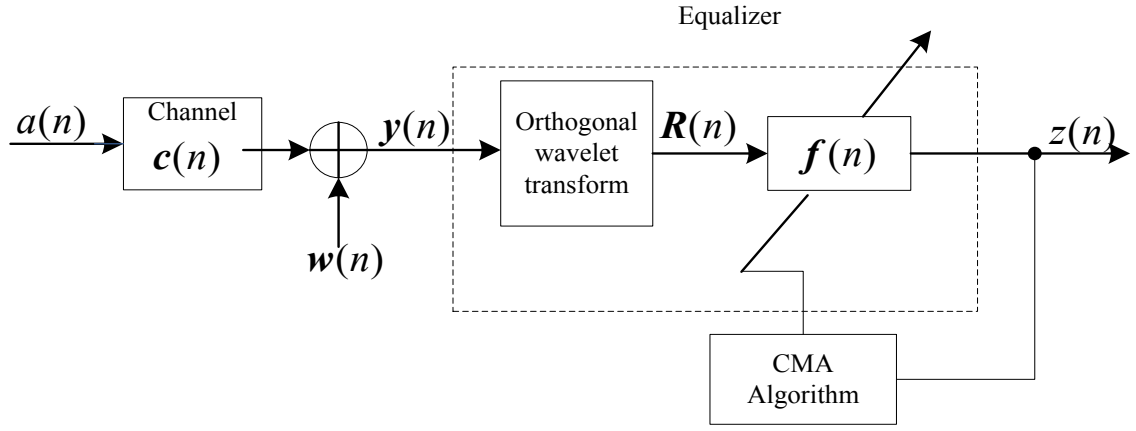

Fig. (1). Adaptive algorithm based on orthogonal wavelet transform.

$R_{2}=\frac{E\left(|a(n)|^{4}\right)}{E\left(|a(n)|^{2}\right)}$

$z(n)=\boldsymbol{f}^{H}(n) \boldsymbol{R}(n)$

$\boldsymbol{R}(n)=\boldsymbol{Q y}(n)$

$\boldsymbol{f}(n+1)=\boldsymbol{f}(n)+\mu \hat{\boldsymbol{R}}^{-1}(n) \boldsymbol{R}(n) e(n) z^{*}(n)$

$e(n)=z(n)\left[|z(n)|^{2}-R_{2}\right]$

In the formula, $\boldsymbol{Q}$ shows the orthogonal wavelet transform matrix, superscript $H$ shows conjugate transpose, $e(n)$ shows the error function, and $\mu$ shows the step length,

$\hat{\boldsymbol{R}}^{-1}(n)=\operatorname{diag}\left[\sigma_{j, 0}^{2}(n), \sigma_{j, 1}^{2}(n), \mathrm{j}^{-}, \sigma_{j, k_{J}}^{2}(n), \sigma_{J+1,0}^{2}(n), \mathbf{j}^{-} £-\sigma_{J, k_{J}}^{2}(n)\right]$, Among them $\sigma_{j, k_{J}}^{2}(n), \sigma_{J+1, k_{J}}^{2}(n)$ respectively for $r_{j, k}(n)$, $s_{J, k}(n)$ of the average power estimation, by the following recursive formula get:

$\left\{\begin{array}{l}\hat{\sigma}_{j, k}^{2}(n+1)=\beta \hat{\sigma}_{j, k}^{2}(n)+(1-\beta)\left|r_{j, k}(n)\right|^{2} \\ \hat{\sigma}_{J+1, k}^{2}(n+1)=\beta \hat{\sigma}_{J+1, k}^{2}(n)+(1-\beta)\left|\mathrm{s}_{J, k}(n)\right|^{2}\end{array}\right.$

However, WTCMA algorithm has easy convergence to the local minimum points but the performance of channel fading resistance is poor. In order to overcome WTCMA's performance defects and improve the adaptive performance, combination of parallel genetic algorithm, the space diversity and orthogonal wavelet adaptive algorithm is required.

\section{THE BALANCE TECHNOLOGY BASED ON SPACE DIVERSITY}

\subsection{Spatial Diversity Equalizer}

Space diversity also called the antenna diversity is the use of more diversity form in communication. In simple terms, it is the use of multiple receiving antenna to receive signals [5]. Because the decline of each primitive received signal can be regarded as independent of each other, and all channel colleagues fading probability is very low, the space diversity technology is one of the effective ways to eliminate the decline. The space diversity technology is applied to the adaptive equalizer, which can effectively overcome the channel fading and inter-symbol interference. Spatial diversity equalizer (Spatial Diversity Equalizer, SDE) principle is shown in Fig. (2).

In Fig. (2), $\boldsymbol{a}(n)$ is independent with the distribution of the emission signal sequence; $\boldsymbol{c}_{l}(n)$ is the NO.l road channel of impulse response vector;

$\boldsymbol{w}_{l}(n)$ is the NO.l road of gaussian white noise

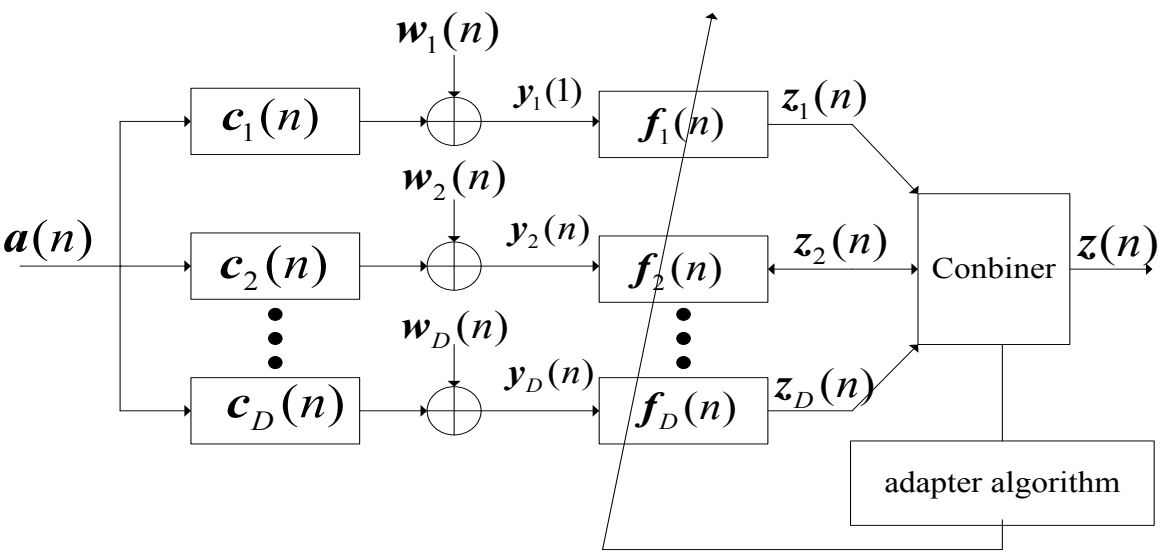

Fig. (2). Spatial diversity equalizer structure. 
sequence; $\boldsymbol{y}_{l}(n)$ shows the NO.l road of input signal vector; $\boldsymbol{f}_{l}(n)$ is the NO.l road of the equalizer's weight vector; among them $M_{f}$ shows the length of the equalizer; $\boldsymbol{z}_{l}(n)$ is the NO. $l$ road of the output of the equalizer; among them, $\boldsymbol{z}(n)$ shows combined output signal.

Fig. (2) shows that the adaptive spatial diversity in each branch is made up of sub-channels and the sub-adaptive device. Signal was received through the sub-channel and the sub-equalizer to merge processing, merging processing methods have a choice to merge, maximal ratio combining and equal gain merger. Among them, equal gain merger is the most easy to implement, so this article will use equal gain merger method.

\subsection{The Adaptive Algorithm of Space Diversity Based on Equal Gain Merger}

Two paths of equal gain merger of space diversity adaptive device structure are shown in Fig. (3). According to the Fig. (3), two paths of the equal gain spatial diversity equalizer contain two prior to filter (each path set a filter) $\boldsymbol{f}_{\mathrm{F}}^{(i)}(n)$ and a rear filter $\boldsymbol{f}_{\mathrm{B}}(n)$. Three filters weight vector is united by the adaptive algorithm based on decision feedback adjustment, then

$$
\begin{aligned}
& z(n)=\sum_{i=1}^{2} y^{(i)}(n)\left[\boldsymbol{f}_{\mathrm{F}}^{(i)}(n)\right]^{\mathrm{T}}-\hat{\boldsymbol{a}}(n-1) \boldsymbol{f}_{\mathrm{B}}(n) \\
& \boldsymbol{f}_{\mathrm{F}}^{(1)}(n+1)=\boldsymbol{f}_{\mathrm{F}}^{(1)}(n)-\mu_{\mathrm{F}} z(n)\left[|z(n)|^{2}-R^{2}\right]\left[\boldsymbol{y}^{(1)}(n)\right]^{*} \\
& \boldsymbol{f}_{\mathrm{F}}^{(2)}(n+1)=\boldsymbol{f}_{\mathrm{F}}^{(2)}(n)-\mu_{\mathrm{F}} z(n)\left[|z(n)|^{2}-R^{2}\right]\left[\boldsymbol{y}^{(2)}(n)\right]^{*} \\
& \boldsymbol{f}_{\mathrm{B}}(n+1)=\boldsymbol{f}_{\mathrm{B}}(n)-\mu_{\mathrm{B}} z(n)\left[|z(n)|^{2}-R^{2}\right] \hat{\boldsymbol{a}}^{*}(n-1)
\end{aligned}
$$

Take the above equal gain merger of space diversity adaptive device apparatus for EG - SDE.

\section{INTRODUCING PARALLEL GENETIC OPTIMI- ZATION OF SPATIAL DIVERSITY WAVELET ADAPTIVE ALGORITHM}

\subsection{Parallel Genetic Algorithm}

Parallel genetic algorithm (Parallel Genetic Algorithm, PGA) is suitable for complex constrained optimization problem of global optimization ability. Parallel genetic algorithm mainly has three categories: (1) the master-slave parallel genetic algorithms; (2) coarse-grained degree parallel genetic algorithm; and (3) fine-grained degree parallel genetic algorithm [6]. Among them, coarse-grained parallel genetic algorithm distributes several sub populations to their corresponding processor, each processor is not only independent of calculation fitness, but also independent of selection, restructure crossover and mutation operation, and regularly send each other the best individual fitness, thus speeding up to meet the requirements of the termination conditions. It is a parallel genetic algorithm of the most widely used currently [7].

Migration is a parallel genetic algorithm introduced into a new operator, which is to point to in the course of evolution of neutron population exchange between individual processes. The general migration method is to send the best individual in the group to other subgroups, as migration can accelerate better individuals in the group communication, improve convergence rate and precision of the solution [8]. Compared with the single population, it needs only a small number of individual evaluation calculation workload. As a result, even with a single processor computer in serial way (pseudo parallel) to implement parallel algorithm can also produce good results [9]. Therefore, use of migration operator makes the parallel algorithm more suitable for global optimization, and small amount of calculation is required.

The most basic migration model is ring topology model and individual transfer occurs only on the adjacent subpopulation. In the adjacent transfer model, the transfer occurs only in the close neighbor set. The model is shown in Fig. (4).

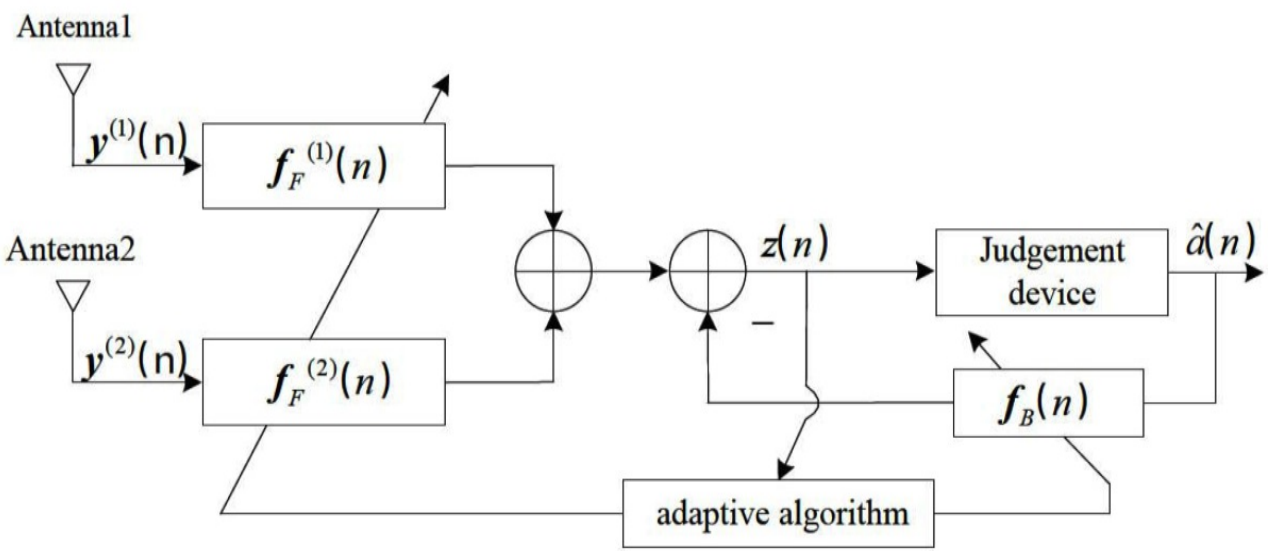

Fig. (3). Equal gain merger of space diversity adaptive device structure. 


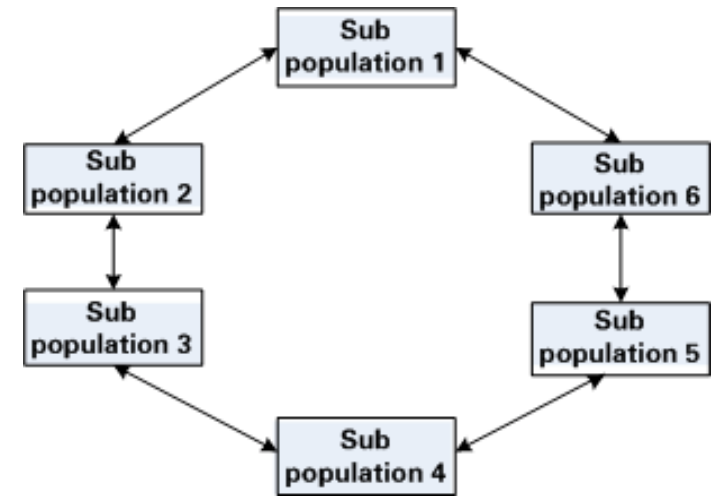

Fig. (4). Ring topology model figure.

This study utilizes the combination of the coarse-grained degree parallel genetic algorithm based on migration of ring typologies, space diversity, orthogonal wavelet transform and adaptive algorithm, with parallel genetic algorithm having fast convergence speed, the global optimal solution, and the orthogonal wavelet transform to reduce the correlation of signal and noise etc. For space diversity, adaptive algorithm is optimized by equal gain merger to get space diversity orthogonal wavelet adaptive algorithm based on parallel genetic optimization.

\subsection{The Orthogonal Wavelet Adaptive Algorithm of Space Diversity based on Parallel Genetic Optimization}

By reference to the parallel genetic algorithm and the orthogonal wavelet transform based on equal gain merger of adaptive algorithms, get orthogonal wavelet adaptive algorithm of spatial diversity based on parallel genetic optimization. This algorithm has high convergence rate and precision of solution, and can get the global optimal solution. Three paths of orthogonal wavelet adaptive algorithm of space diversity based on parallel genetic optimization principle are shown in Fig. (5).

In Figure 5, $n$ shows the time sequence, $\boldsymbol{a}^{(i)}(n)$ shows the NO. $i$ road launch signal, $c^{(i)}(n)$ shows the NO. $i$ road channel impulse response, $\boldsymbol{w}^{(i)}(n)$ shows the NO. $i$ road channel output end additive gaussian white noise, $\boldsymbol{f}_{F}^{(i)}(n)$ shows the NO. $i$ road pre-equalizer weight vector, $\boldsymbol{y}^{(i)}(n)$ shows the NO. $i$ road output signal, $\boldsymbol{y}(n)$ shows signal after equal gain merger, $\boldsymbol{z}(n)$ shows the output signal, $\boldsymbol{f}_{B}(n)$ shows the orthogonal wavelet adaptive device weight vector, $\boldsymbol{J}^{(i)}\left(f_{m}\right)$ shows the NO. $i$ road parallel genetic algorithm's son population of cost function.

This algorithm contains three forward equalizer (each path set has an equalizer), each branch of the equalizer weight vector as the decision variables of parallel genetic algorithms, design initial population Chrom $^{(i)}=\left[\boldsymbol{f}_{1}, \boldsymbol{f}_{2}, \cdots, \boldsymbol{f}_{M}\right]$, of which $M$ is individual number. In the population of every individual, $\boldsymbol{f}_{m}(1 \leq m \leq M)$ corresponds to a balancer weight vector. This paper constructs the initial population based on the characteristics of parallel genetic algorithms, namely there are three son populations, each son population corresponding to a path. Considering the characteristics of adaptive, weight vector of each module value will be less than 1 , so will the search as the $[0,1]$.

Assume that the received signal sequence of length for $N$ uses time average instead of the statistical average, formula (1) often shows constant model algorithm of the cost function can be calculated by the next formula:

$J^{(i)}\left(\boldsymbol{f}_{m}\right)=\frac{\sum_{j=1}^{N}\left(R-\left|y_{m}^{(i)}(j)\right|^{2}\right)^{2}}{N}$

In the formula, $m$ shows the equalizer weight vector of individual serial number, $N$ shows the received signal sequence length for each generation, $i$ shows the son population number, $y_{m}^{(i)}(j)$ shows each equalizer weight vector of the output signal of individual by each path. The above formulas function as the objective function of parallel genetic algorithms and solve their minimum value, and obtain the best individual optimal weights coefficient of adaptive algorithm. Due to the $J^{(i)}\left(\boldsymbol{f}_{m}\right)>0$, the individual fitness function is given as:

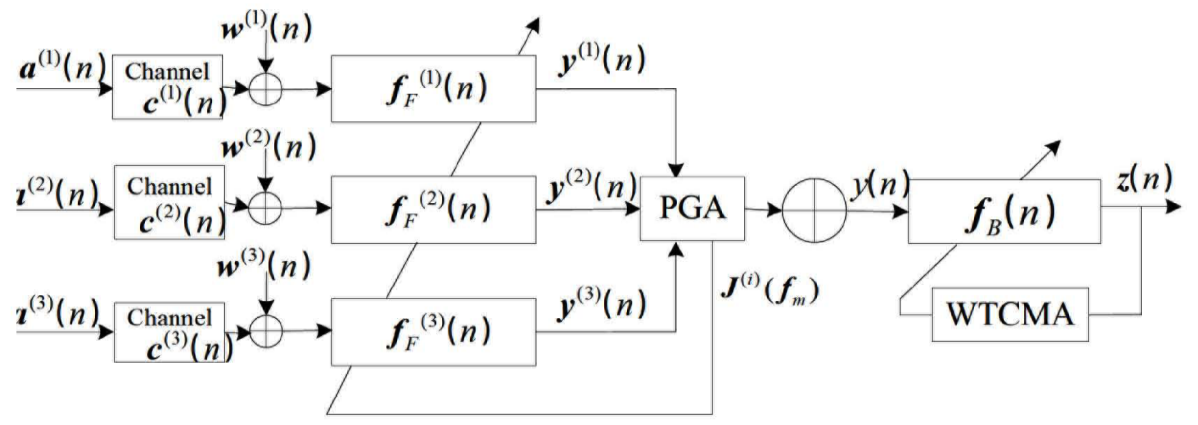

Fig. (5). Based on parallel genetic optimization and orthogonal wavelet adaptive algorithm of space diversity principle block diagram. 
$F_{i t}^{(i)}(m)=\frac{1}{J^{(i)}\left(\boldsymbol{f}_{m}\right)}$

This paper uses the coarse-grained degree parallel genetic algorithm based on migration of ring topology. Each branch of the equalizer weight vector functions as a sub population of parallel genetic algorithm. On entering the parallel genetic algorithm, each sub population is assigned to each processor independent fitness calculation, selection, crossover and mutation operations, and every generation of a ring topology operation on migration, each sub population has copies with the highest fitness individuals to adjacent sub populations, and replaces its lowest fitness of individuals. This can avoid algorithm premature convergence, and can improve the convergence speed. Meeting after termination conditions of genetic algorithm, each branch of output signal after equal gain merger, input rear equalizer, and the equalizer weight vector by the adaptive algorithm adjustment based on orthogonal wavelet transform, this plays an important role in small range search and search speed. The orthogonal wavelet adaptive algorithm based on parallel genetic optimization and space diversity makes full use of parallel genetic algorithm, space diversity and orthogonal wavelet adaptive algorithms respective advantages, to obtain better adaptive effect.

\section{THE COMPUTER SIMULATION EXPERIMENT}

In order to test the performance of the PGA-SDWTCMA algorithm compared with the WTCMA and the EG-SDE as the objects, having the computer simulation experiments. In this paper, the genetic algorithm program used a genetic algorithm toolbox (GATBX) based on Matlab. Channel used mixed phase underwater acoustic channels $c_{1}=[0.3132,-0.104,0.8908,0.3132], \quad$ minimum phase underwater acoustic channels $c_{2}=[0.8264,0.1653,0.1653]$ and two size underwater acoustic channel $c_{3}=[-0.35,0,0,1]$. Emission signal was16QAM, signal to noise ratio was $20 \mathrm{DB}$, and the length of the equalizer was 16 .

The algorithm parameter selections are as follows: WTCMA used the channel $c_{1}$, the initial weight vector of the NO.4 tap took 1, other took 0, step length took 0.000015; EG-SDE used the channel $c_{1}$ and $c_{2}$, among them CMA's initial weight vector of the NO.4 tap took 1 , other took 0 , step length took 0.000005 ;

PGA-SD-WTCMA used the channel $c_{1}, c_{2}$ and $c_{3}$, in the algorithm of WTCMA's parts initial weight vector of the NO.5 tap took 1 , other took 0 , step length took 0.00003 . The sub-population size took 20 , crossover probability took 0.7 , and the mutation probability took $1 / 16$, taking every generation migration, termination conditions for evolution to the tenth generation.

The orthogonal wavelet change adopted $\mathrm{Db} 4$ wavelet in the WTCMA and the PGA-SD-WTCMA, decomposition layer number is $2, \beta$ took value for 0.99 , and power initialization value is 10 ;
The simulation results are shown in Fig. (6).

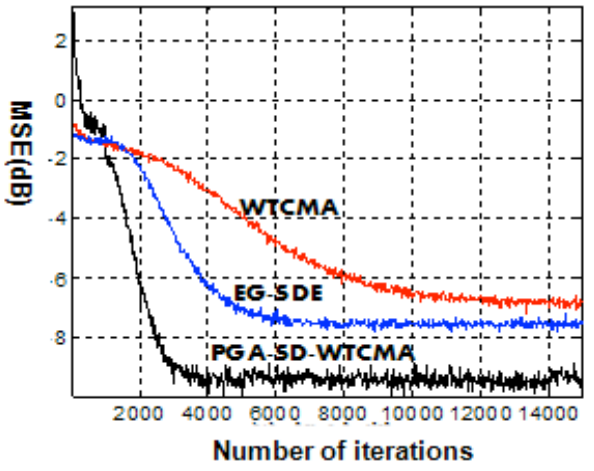

(A) The mean square error of curve

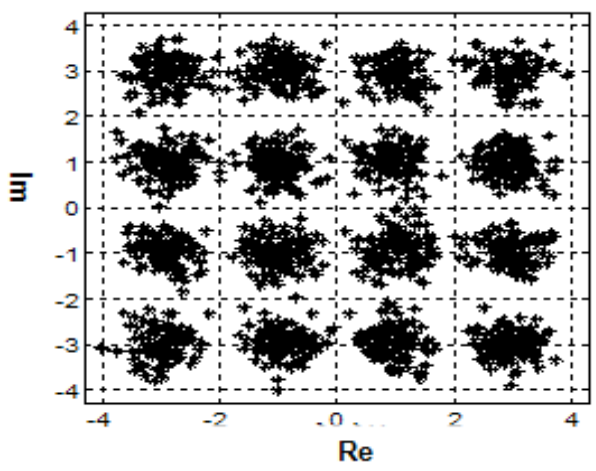

(B) WTCMA output signal

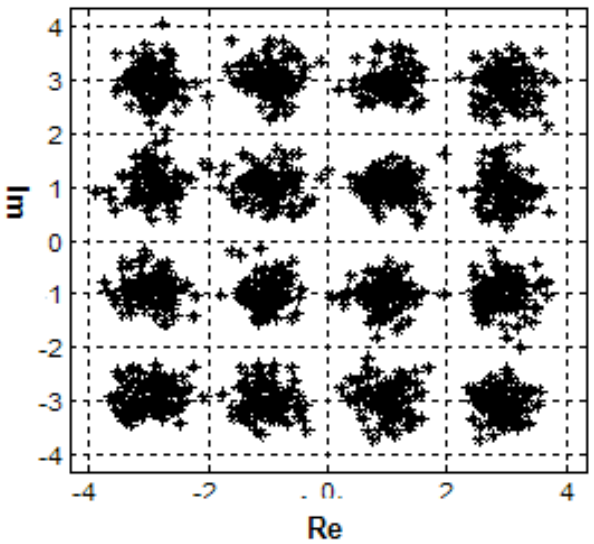

(C) EG-SDE output signal

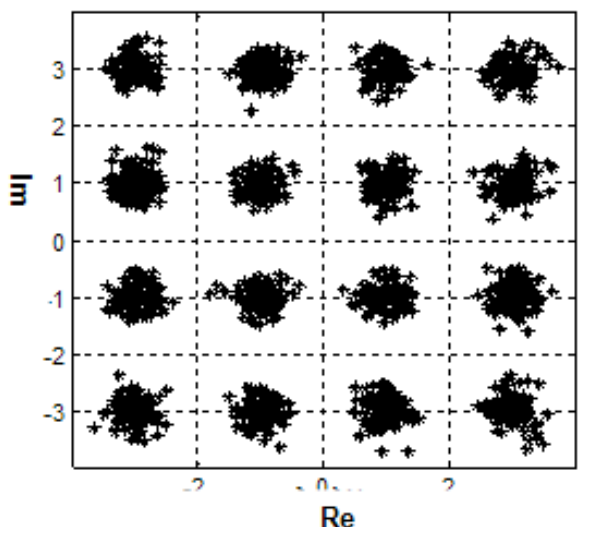

(D) PGA-SD-WTCMA output signal

Fig. (6). The computer simulation results. 
Fig. (6A) shows that the convergence rate of the PGASD-WTCMA than the WTCMA and the EG-SDE increased nearly 7000 times and 2500 times respectively, and the mean square error is decreased nearly $3 \mathrm{~dB}$ and $2 \mathrm{~dB}$, respectively.

Fig. (6B-D) shows that after the PGA-SD-WTCMA optimization of equalizer output data error than the WTCMA and the EG-SDE decreased significantly, the constellation diagram is shown more clearly.

The above results show that the orthogonal wavelet adaptive algorithm based on spatial diversity by parallel genetic algorithm optimized can effectively accelerate the convergence speed and reduce the mean square error (MSE), hence the performance is improved significantly.

\section{CONCLUSION}

In order to overcome the influence of the multipath effect and channel fading, the combination of the space diversity, parallel genetic algorithm, orthogonal wavelet transform and adaptive algorithm proposed the orthogonal wavelet adaptive algorithm based on parallel genetic optimization and space diversity. The algorithm makes full use of space diversity, decreasing the influence of the multipath effect, using parallel genetic algorithm for space diversity's each branch of the equalizer weight vector optimization, thus accelerating the algorithm convergence speed and global convergence by using auto-correlation based on orthogonal wavelet transform to reduce the signal so that the algorithm achieves good performance. The computer simulation results verify the effectiveness of the algorithm.

\section{CONFLICT OF INTEREST}

The authors confirm that this article content has no conflict of interest.

\section{ACKNOWLEDGEMENTS}

This work was supported by the General Program of the Natural Science Fund of Hubei Province, China under Grant No. 2014CFB916 and the General Program of the Natural Science Fund of Hubei Province, China under Grant No. 2014 CFB290.

\section{REFERENCES}

[1] Y. K. Alain, and F. Gerard, "Blind equalization of nonlinear channels using a tensor decomposition with code /space /time diversities," Press Signal Processing, vol. 89, pp. 133-143, 2009.

[2] Y. Han, "The research of adaptive device design and algorithm simulation based on wavelet transform", MS thesis, Anhui University of Science and Technology, 2007.

[3] Y. Li, K. Li, and X. Lu, "The Neural network adaptive algorithm based on genetic optimization," Journal of China's North University, vol. 30, no. 2, pp. 137-142, 2009.

[4] X. Wang, and I. Caol, "Genetic algorithm (ga) theory, application and software implementation," Xi 'an Jiaotong University Press, China, 2003.

[5] X. Din, The orthogonal wavelet adaptive algorithm based on diversity technology, MS thesis, Nanjing Information Engineering University, 2010.

[6] Y. Chen, and Y. Tian, "An improved parallel genetic algorithm to solve TSP," IEEE Transactions on Electron Devices, vol. 31, no. 7, pp. 112-119, 2014.

[7] S. Xue, S. Guo, and D. Bai, "The analysis and research of parallel genetic algorithm," In: $4^{\text {th }}$ International Conference, Wireless Communications, Networking and Mobile Computing, 2008, pp. 1-4.

[8] Z. Konfrst, "Parallel genetic algorithm: advances, computing trends, applications and perspectives," In: Parallel and Distributed Processing Symposium, 2004, pp. 162-169.

[9] M. Rebaudengo, and M.S. Reorda, "An experiment analysis of the effects of migration in Parallel Genetic Algorithms," In: Parallel and Distributed Processing, 1993, pp. 232-238.

Received: September 16, 2014

Revised: December 23, 2014

Accepted: December 31, 2014

(C) Xiaoping et al.; Licensee Bentham Open.

This is an open access article licensed under the terms of the Creative Commons Attribution Non-Commercial License (http://creativecommons.org/licenses/by-nc/4.0/) which permits unrestricted, non-commercial use, distribution and reproduction in any medium, provided the work is properly cited. 